\title{
EVALUATION OF WORKFLOW MANAGEMENT SYSTEMS - A META MODEL APPROACH
}

\author{
Michael Rosemann, Michael zur Muehlen \\ Department of Information Systems \\ Westfaelische Wilhelms-Universitaet Muenster \\ Steinfurter Str. 107 \\ D-48149 Muenster \\ Germany \\ \{ismirolismizu\}@wi.uni-muenster.de \\ Phone +49-251-83-38100 \\ Fax +49-251-83-38109 \\ Correspondence: Dr. Michael Rosemann \\ July 22 - October 31, 1998: \\ Queensland University of Technology \\ School of Information Systems \\ 2 George Street \\ GPO Box 2434 \\ Brisbane QLD 4001
}

\begin{abstract}
The automated enactment of processes through the use of workflow management systems enables the outsouring of the control flow from application systems. By now a large number of systems, that follow different workflow paradigms, are available. This leads to the problem of selecting the appropriate workflow management system for a given situation. In this paper we outline the benefits of a meta model approach for the evaluation and comparison of different workflow management systems. After a general introduction on the topic of meta modeling the meta models of the workflow management systems WorkParty (Siemens Nixdorf) and FlowMark (IBM) are compared as an example. These productspecific meta models can be generalized to meta reference models, which helps to specify a workflow methodology. Exemplary, an organisational reference meta model is presented, which helps users in specifying their requirements for a workflow management system.
\end{abstract}

\section{BASICS OF WORKFLOW MANAGEMENT SYSTEMS}

Similar to the transfer of data management away from application systems toward database management systems the transfer of control flow enabled by workflow management systems has a profound meaning for the future architecture of information systems. JoOsTEN defines a workflow management system (with reference to the workflow management coalition (WfMC) ${ }^{10}$ ) as "[...] a system that defines, manages and executes workflows through the execution of software whose order of execution is driven by a computer representation of the business process logic."11

Workflows 12 are processes - temporal and logical sequences of functions that are necessary to perform operations on economically relevant objects - with automated transitions, namely processes, whose control logic lies within the control of an information system. Every workflow is based on a process model that has been enhanced with additional object types (eg. data structures) and attributes (eg. start conditions) that allow its automation, the so called workflow model. Workflow models are usually described using directed graphs whose knots represent (elementary or composite) functions. From the point of view of the workflow management system elementary functions are encapsulated, ie. the workflow management system has an external view on the automated or manual execution of the functions but is not concerned with the execution of the functions themselves.

\footnotetext{
10 Cf. WfMC (1996).

11 Joosten (1995), p. 4.

12 For a variety of interpretations of the term workflow cf. e.g. Georgakopoulos, Hornick, Sheth (1995),
}

pp. $122 f$. 
By now a large number of commercial workflow management systems are available. These systems differ with regard to their efficiency due to their distinct historical background. ${ }^{13}$ The selection of an appropriate workflow management system demands an efficient selection process. During the traditional process of software evaluation the main focus lies on technical aspects, such as reliability, useability, maintainability and adaptability ${ }^{14}$ as well as on economic aspects of the systems analysed. The use of criteria catalogues represents the common practice of software evaluation. However, they fail during the evaluation of modeling methods, because they do not reflect the vast number of modeling alternatives offered by a method. The complexity of these alternatives can be reduced by the formalisation of the method's description.

In the next sections we will analyse how meta models can help (pre-)selecting an appropriate workflow management system. To begin with, we discuss meta models in general and meta data models in particular. In the following chapter the product individual meta data models of WorkParty (SNI) and FlowMark (IBM) ${ }^{15}$ are presented. The comparison and generalisation of the two meta data models leads to a generic organisational reference meta model, which can serve as a benchmark for system specific meta data models. The analysis performed allows us to draw some substantial conclusions. The discussion of this approach is the subject of the last section.

\section{DEFINITION AND INTENTION OF META MODELS}

\section{Characteristics and Occurrences of Meta Models}

A model is an immaterial representation of a relevant part of the real world. It is created for the purpose of a subject, relates two systems and therefore consists of three components: ${ }^{16}$

- The object system represents the subjective interpretation of a selected part of the real world (universe of discourse) including the relevant part of the environment.

- The model system represents the subjective image of the object system. A syntax (also: notation, language) is needed to create the model system.

- The projection formulates the relationship between the object system and the model system. The complexity of the real world, that is generated by the number of real world elements and relationships, is reduced by eliminating irrelevant elements. The variety of real world elements and relationships is controlled by clustering elements and relationships (type generation).

If a model system M1 represents the object system of a model system M2, the model system M2 represents the meta model system of the object system M1 is based upon. ${ }^{17}$ Because of this degree of abstraction a meta model can be seen as "a design framework, that describes the basic model elements and the relationships between the model elements as well as their semantic. This framework also defines rules for the use and specialisation of model elements and relationships." 18

In the context of information modeling we distinguish meta models describing a notation and meta models describing a procedure. ${ }^{19}$ Meta data models characterise notations that can be used for information modeling purposes. Meta process models describe the modeling process using a specific method. Every meta model is based upon another

13 Cf. Joosten (1996), p. 2: "The [...] field of workflow management suffers from confusion caused by weakly defined concepts and a lack of consensus about the way in which these concepts are used."

14 Cf. Dunn (1993); Kazman et al. (1995).

15 Cf. Leymann, Altenhuber (1994).

16 Cf. Hars (1994), pp. 7-11.

17 Cf. Hars (1994), p. 11. cf. Nissen et al. (1996), p. 38.

18 Cf. Ferstl, Sinz (1994), p. 86; Steele, Zaslavsky (1994), p. 317: "Meta models [...] might be expressed using one or more modelling techniques, that in combination are able to adequately model all relevant aspects of any given modelling technique."

19 Cf. Saeki (1996). 
meta model, which can be of the same kind, eg. the notation of the entity relation diagram may be explained using another entity relation diagram. If the similarities of a number of meta models are consolidated in one universal model that also claims a high degree of semantic quality this model is called a reference meta model and specifies the common methodology.

In the past a number of different meta models for workflow management systems have been introduced. In most cases these models are reference meta models. Examples for such reference meta models can be found in DERUNGS (1997), GALLER (1997), JABLONSKI, BUSSLER (1996), JOOSTEN (1996) and RUPIETTA (1997).

The main objective of this paper is the evaluation of product individual meta models as well as the discussion of the results delivered by a comparison of these (detailed) meta models. The integration of the presented product individual meta models into an - inductively generated - reference meta model is outlined further on.

\section{Description of the Notation}

The meta data models in this paper are designed using extended Entity-Relationship Models. ${ }^{20}$ An extension of this method are variable-based integrity constraints ${ }^{21}$, which allow instance specific definitions of semantic relationships (eg. exclusive-OR) between cardinalities. This is achieved by replacing the cardinalities with variables and relating the cardinalities in separate integrity constraints. Although variable based integrity constraints raise the complexity of the meta data models, they are useful as they enable the description of specific rules of a certain method. Furthermore, generalisations are established for reasons of clarity, eg. groupings of all information objects that are relevant for the staff resolution. For every generalisation a description of the disjointness constraint (d (disjoint) or $n$ (not disjoint)) and the completeness constraint ( $\mathrm{p}$ (partial) or $\mathrm{t}$ (total)) is given. ${ }^{22}$

\section{COMPARISON OF META MODELS}

\section{Conflict Identification and Resolution}

Prior to the evaluation of meta models it has be made sure that all possible conflicts between the models have been resolved. Three major kinds of conflicts can be identified: ${ }^{23}$

Naming conflicts occur, if the naming conventions of the models to be compared show synonyms or homonyms. These are the most common conflicts during the evaluation of meta data models. A synonym can be found if two or more linguistic expressions share the same meaning. A homonym results of the ambiguity of a linguistic element. In its context the meaning of a homonym is unique, but not because of its notion alone.

The determination of synonyms requires an analysis of different terms with identical meaning and associations. Hints about potential synonyms can be found by tracing similar structures embedding information objects with different names (concept likeness) ${ }^{24}$. Homonyms contradict the clarity of a model, because the notion of the term can be determined depending on the user and the context, but not in general. A potential indicator for the identification of homonyms in meta models are information objects with the same name that are embedded in different structures (concept unlikeness).

A type conflict can be located, if the same fact is represented semantically correct in two models through different methodical concepts. Structural conflicts arise if the meta models to be integrated depict the same facts using different semantics. This is a violation of the semantic correctness. Structural conflicts often arise if different people are involved in the modeling process. Type and structural conflicts are not relevant at this point, because the meta data models to be compared and to be evaluated were created by the same group of people.

\section{Objects of a Meta Model Evaluation}

20 Cf. Chen (1976).
$21 \quad$ Cf. Rosemann (1996).
22 Cf. Elmasri, Navathe (1995), p. 618. They use the term overlapping instead of not disjoint.
23 Cf. Hars (1994), pp. 194-206.
24 Cf. Batini, Lenzerini (1984); Batini, Ceri, Navathe (1992), p. 124.


When two conflict-free meta data models are compared, the following information objects can provide useful information:

\section{Entity types}

The comparison of the number and kind of entity types provides the most essential information for the comparison of meta data models. Within a given degree of abstraction the adaptability of a workflow management system increases with the number of entity types the meta data model consists of. The validity of a qualitative and quantitative rating of entity types is determined by the degree of detail of the models in the first place.

\section{Relationship types}

Another metric concerning the flexibility of a workflow management system are the relationship types. The flexibility of a workflow management system increases with the number of relationship types, if the number and kind of entity types stay the same. An example for different relationship types can be found in the organisational model of a workflow management system, where the relations between actors, organisational units and roles differ between the systems.

\section{Cardinalities}

The cardinalities provide useful information about the design options a specific system provides. A workflow management system, that allows a $(0,1)-(0, n)$-hierarchy of organisational units, simultaneously prohibits the design of multi-dimensional organisations (eg. matrix-structures). The introduction of variablebased cardinalities enhances the semantic capability of a model, but complicates the comparability of the model.

Attributes

Beyond the elements an ERM usually includes, the comparison of attributes provides further information about the system characteristics a workflow developer can use. Attributes have to be distinguished into attributes used by the system and additional ('free') attributes. User defined attributes allow a higher flexibility than predefined attributes.

\section{Evaluation of the Organisational Meta Models}

Before the meta data models are compared, a brief description of the central entity types is given. Potential naming conflicts have already been resolved during the modeling of the two Entity-Relationship-Diagrams depicted in fig. 1 and 2. For example, the entity type role of WorkParty was renamed position type, while the entity type competence was renamed role in order to avoid a homonym conflict with the corresponding terms of FlowMark. ${ }^{25}$

An organisational position in WorkParty is the domain of a person within an organisational unit and is assigned to exactly one organisational unit. An organisational position can be occupied by one person, but it may be left empty, too. In WorkParty an organisational position is an abstraction of a specific person. Zero, one or several persons can act as a substitute for a specific organisational position. An organisational position can belong to a certain position type. This entity type cannot be found in the meta model of FlowMark.

An organisational unit in WorkParty is a part of the company organisational structure, eg. a department. It is created as a composition of organisational positions or subordinate units. Organisational units can be temporary or permanent, the former are called project units, the latter are called line units and form the core of the company organisational structure. The hierarchical structure of organisational units is restricted to a tree structure. An organisational unit may be associated with several roles and positions and can be the owner of several resources. Similar to WorkParty, an organisation in FlowMark represents an administrative unit of the company organisational structure. However, a person in FlowMark can only be a member of one organisation. An organisation has one specific manager and may have an unlimited number of members with different roles, but at least one (the manager). synonym conflict in the renamed entity type competence and the original entity type role of WorkParty. A correct resolution of these conflicts asks for a new context free term for the entity types concerned. However, the term role has a generally accepted meaning in this context and is used by the WfMC as well. Therefore, in this particular case the conflict resolution is passed up in order to use generally accepted terminology. 
Resources (only in WorkParty) are items that ultimate units of responsibility may dispose in order to fulfil their tasks. Each resource may be assigned to one or more roles. This leads to the definition of access rights for certain persons. Each resource has one specific owner, which may be a person, an organisational unit, or an organisational position. Resources can be organised in an is-part-of-relationship, thus being superior or subordinate to other resources.

Levels (only in FlowMark) represent a hierarchy inside the organisation that need not be identical with the company organisational structure (eg. years of membership or tariff).

When compared directly (fig. 1 and 2), the WorkParty meta model shows a larger number of entity types than the FlowMark meta model, which points to a higher flexibility of modeling different organisational structures. FlowMark misses especially the entity types organisational position and position type. In contrast to this, FlowMark offers two relationship types between the entity types person and organisation or person and role, while WorkParty offers only one relationship type between these entity types.

FlowMark shows more restricted cardinalities than WorkParty. Especially the rigid $(1,1)-(1, n)$-relationship between the entity types person and organisation is expected to be a handicap when modeling organisations. The possibility of assigning several positions to one person in WorkParty enables the modeling of multi-dimensional organisations and shows greater flexibility than the solution provided by FlowMark. Both products show a restricted $(0,1)-(0, n)-$ hierarchy over the entity type organisation. In addition, the substitute-relationship for the entity type person in FlowMark is restricted to only one substitute per person $((0,1)-(0, n)$-relation), while WorkParty allows an unlimited number of substitutes. In WorkParty a person has to be assigned to a position and therefore to a specific organisational unit. This prohibits the modeling of external workers, such as contractors.

The interface between the organisational meta model and the process meta model is provided by an address expression. This entity type enables a dynamic staff resolution at runtime, eg. according to the criteria given in the address expression and the current system users, all persons are determined, that match the given criteria. In general, direct and indirect address expressions can be distinguished. While the former explicitly reference the authorised actors in person, the latter contain criteria like organisational units or positions which are used to determine a subset of authorised persons at runtime. The use of indirect address expressions makes processes more resistant against changes in the organisational model of a company than it would be possible with a direct assignment of persons to activities.

\section{Evaluation of the Process Meta Models}

Both FlowMark and WorkParty provide the user with a graphical modeling method for designing workflow models. In FlowMark workflow models are drawn as graphs with weighted transitions through the explicit design of knots and connectors. WorkParty offers the user pre-defined process components, which provide elementary control flow elements, such as iterations, forks and joins.

\section{The Process Meta Model of WorkParty}

In WorkParty a process is a directed graph that determines the sequence of activities or programs respectively. It consists of a starting activity and one or more ending activities, knots and connectors. The starting activity contains global information about the process that are relevant for all activities. Knots contain activities or programs, connectors determine their sequence. Several ending activities can be used to model alternative endings of a process, the starting activity of a process has to be unique.

An activity is an elementary step in a process. Activities can be manual or they can reference an application program. Furthermore, programs may be associated with activities that are only executed in case of an exception. WorkParty supports ad-hoc activities, ie. at runtime the user may insert an unlimited number of new activities at a specified point, before the modelled process is resumed.

A file is a database used by WorkParty for storing process and activity templates. While a theme file contains all process and activity templates that are related to a specific field of business, a template file is a subset of a theme file and contains process and activity templates for a certain kind of business process. Process files are instances of template files and contain attachments and parameters relevant for the specific processes. 


\section{The Process Meta Model of FlowMark}

A process in FlowMark is similar to a process in WorkParty. In addition to the sequence of the activities the process designer in FlowMark has to specify the flow of data between the activities through the use of data connectors. Each process can be assigned to one process category in order to restrict the number of people authorised to execute the process. Furthermore, a process may contain an address expression that is valid in addition to activity individual address expressions. For every process a process administrator can be defined, who is contacted in case of an exception.

An activity is a single step in a process and corresponds to the activity in WorkParty. Activities contain a start condition and an end condition as well as an address expression for the individual staff resolution. FlowMark supports three kinds of activities:

- Process activities are used to invoke sub processes within a process. It is possible to recursively invoke the same process.

- Program activities are (semi-)automated activities, that invoke an application program during their execution.

- Loop elements are used for modeling iterations. If a single activity shall be executed several times the end condition of the activity can be used as a loop condition. If the iteration shall contain several activities a block has to be used. Blocks are equivalent to sub-processes, but their contents are modelled individually. Bundles are used if a single activity has to be instantiated several times.

Containers are used for storing structured data that is transmitted from one activity to another. Each activity, process and block has one input container and one output container. The contents of a container can be addresses using various APIs (C, REXX, COBOL etc.). 


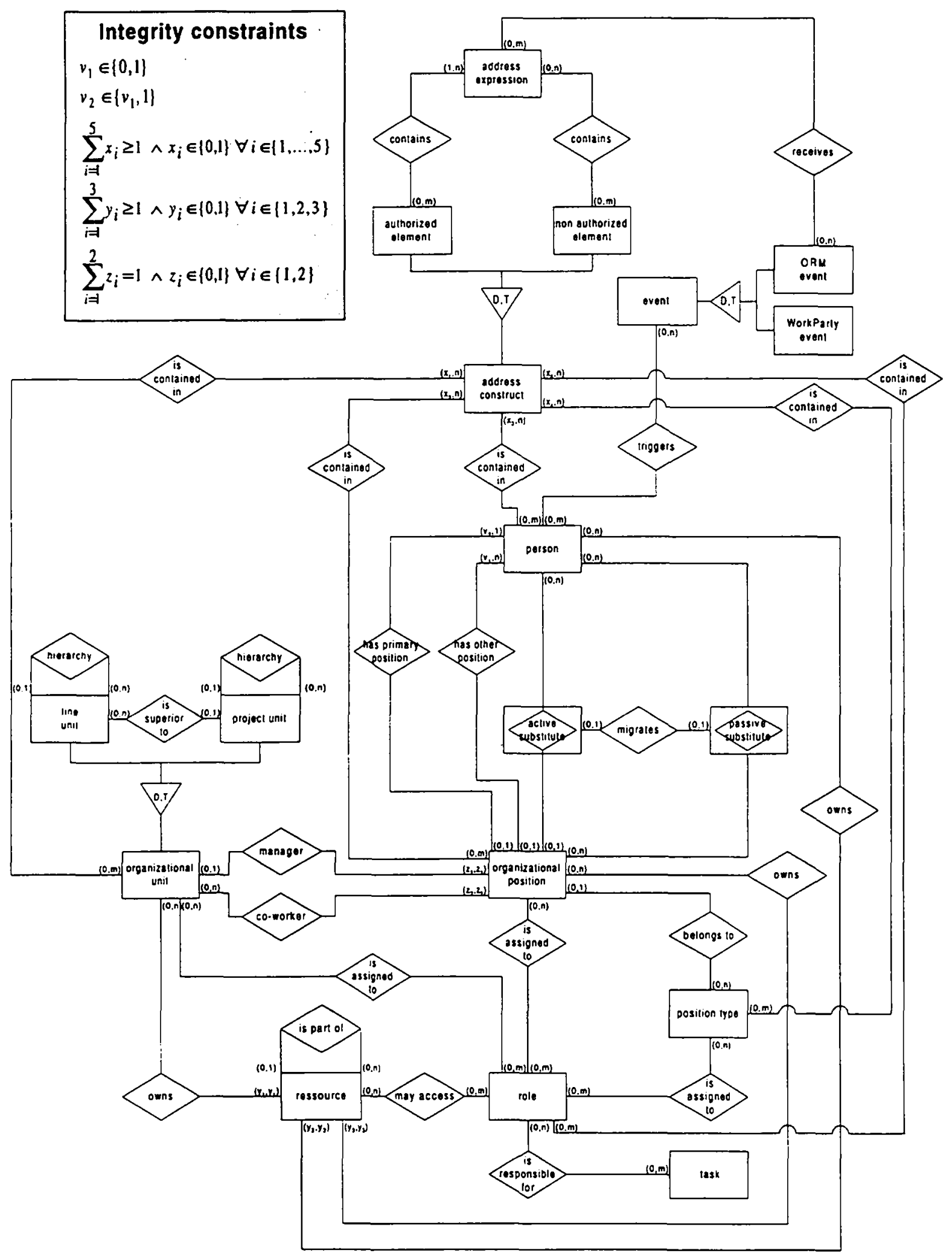

Fig. 1: Organisational meta model of WorkParty 


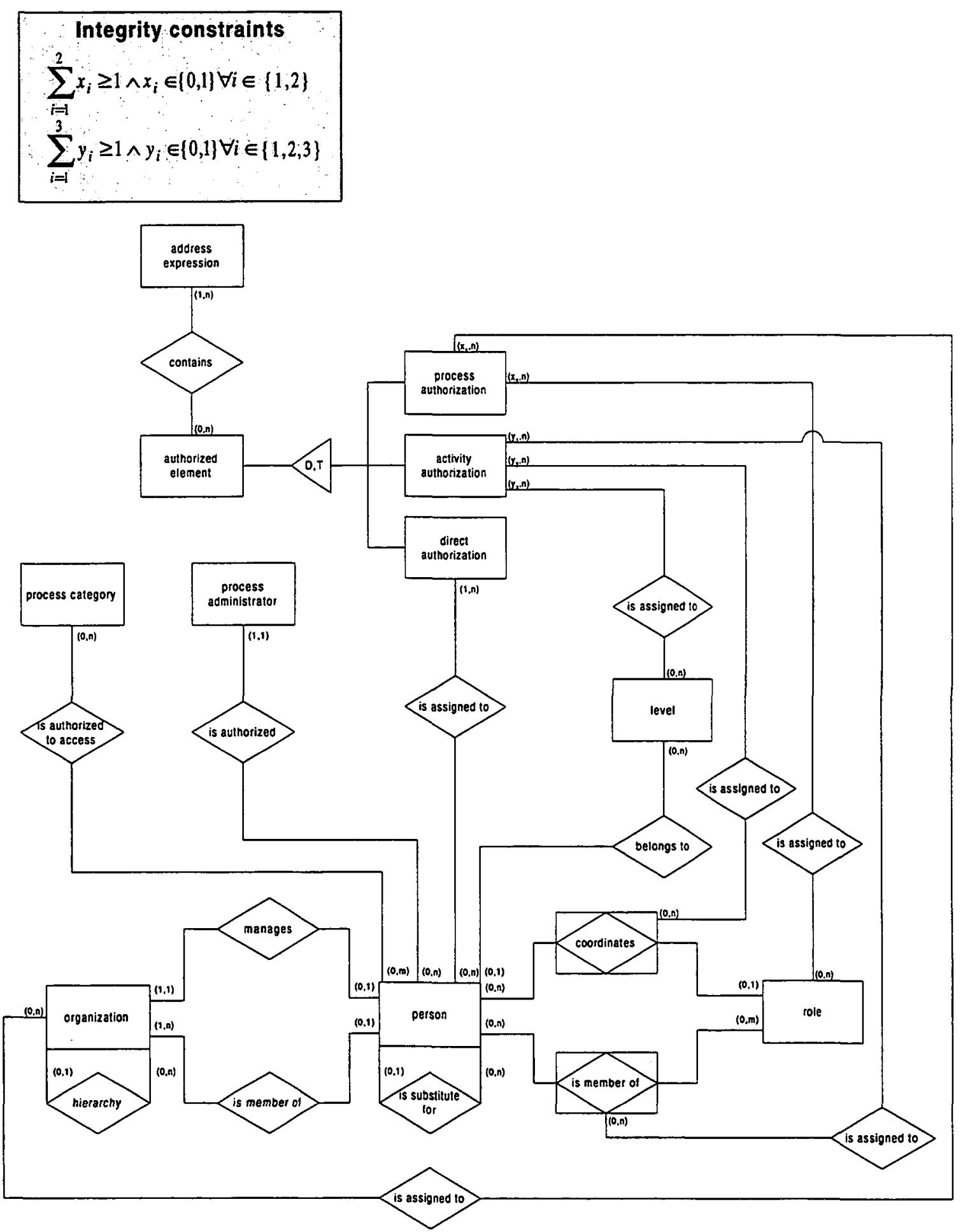

Fig. 2: Organisational meta model of FlowMark 


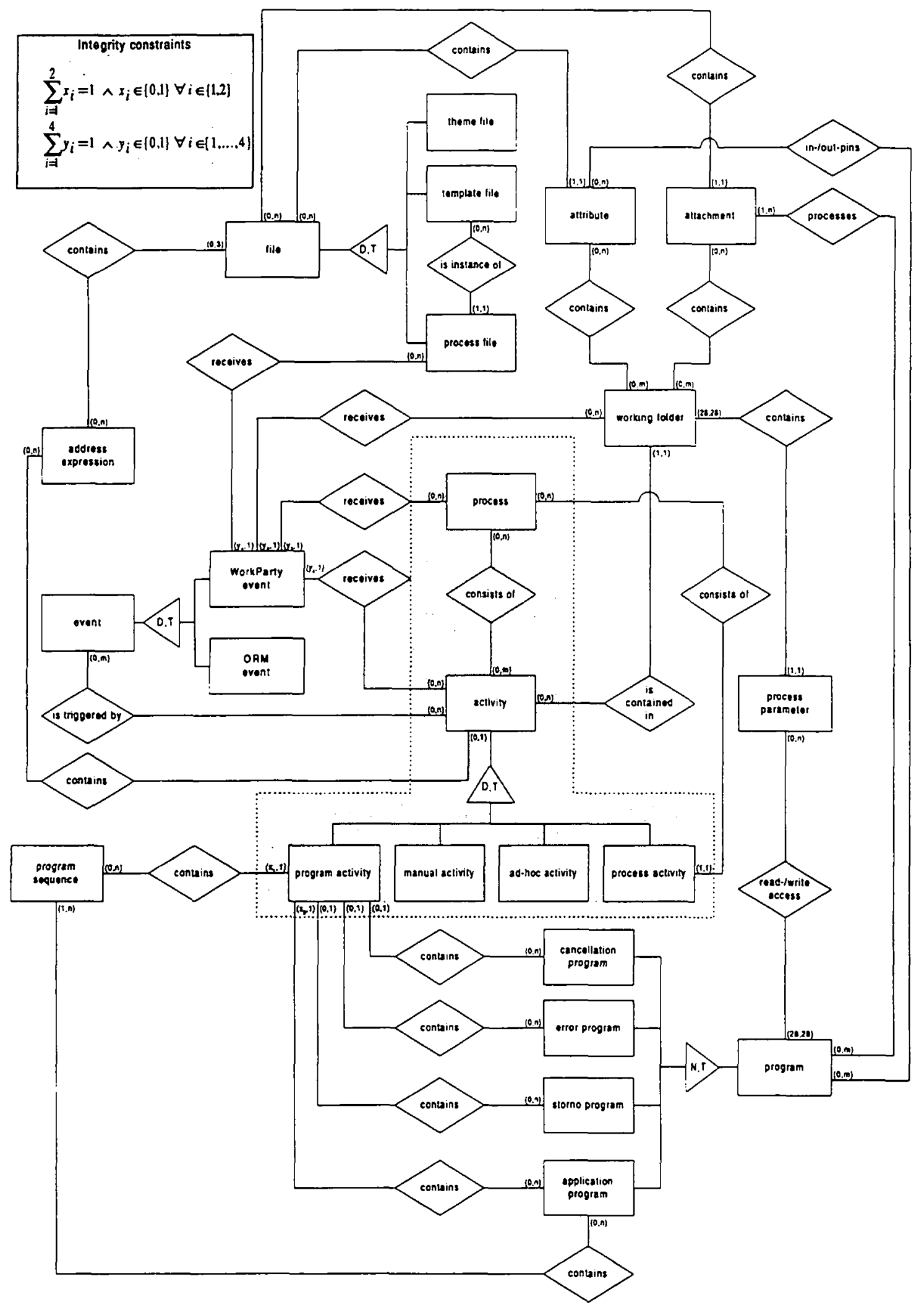

Fig. 3: Process meta model of WorkParty 


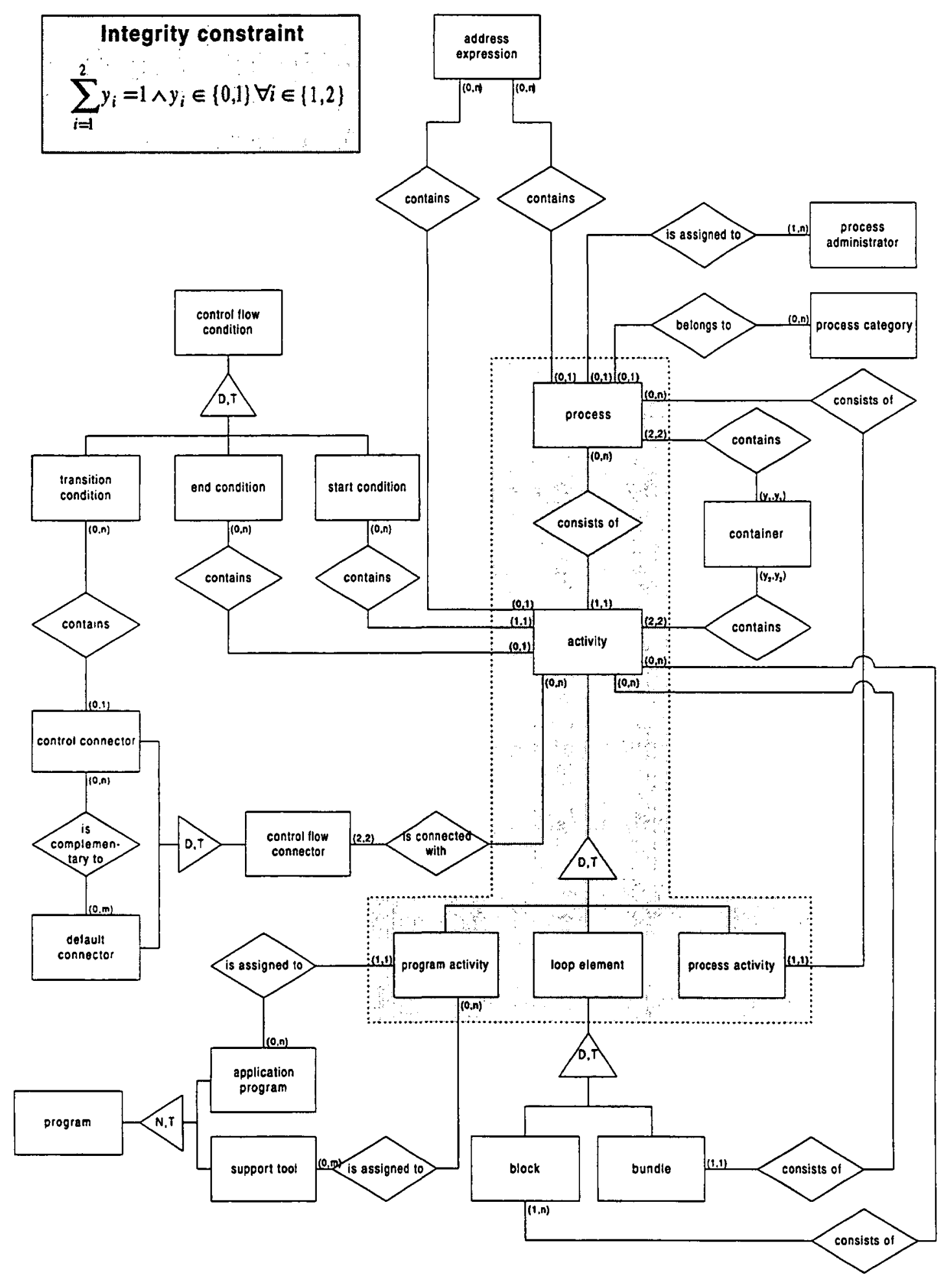

Fig. 4: Process meta model of FlowMark

A connector is a directed edge that determines the control flow and the data flow of a process. Control connectors can have a transition condition that must be fulfilled, otherwise the target activity will not be executed and the path of the connector will be eliminated during a dead path elimination. Default connectors have no transition condition and are followed if no transition condition of the other control connectors evaluates to true. Data connectors are used 
for connecting the input- and output containers of activities. The flow of data in FlowMark need not to be identical with the control flow.

\section{Evaluation of the Process Meta Models}

Similar to the conflict resolution during the comparison of the organisational meta models several conflicts had to be resolved before the process meta models could be compared. For example the entity type process of FlowMark is called sequence in WorkParty. The resolution of conflicts was done by the selection of WfMC-compliant terms. The grey reverse $T$ shapes in figure 3 and 4 indicate characteristic design elements that can be found in the process meta models of different workflow systems.

In FlowMark control and data flow are modelled separately and are therefore visible as distinct elements. In WorkParty the control flow is modelled by customising predefined components while the data flow is not visible in the process model. Instead, the data flow is modelled for each activity individually. The control flow elements available in both systems are not completely represented in the meta models. For example, the bundle activity of FlowMark can be implemented in WorkParty as well, using certain iteration components in combination with optional process branches. Therefore, the semantic power of the meta models cannot be compared without taking the control flow elements into account.

Contrary to the organisational meta model a direct comparison of the number of entity types does not seem reasonable for the process meta models. Because of the different workflow philosophies the mere number of entity types does not lead to conclusions about advantages or disadvantages of a specific system. The selection of the appropriate level of detail is difficult, too, because on the one hand the meta models should not be subject to unlimited growth and on the other hand sufficient aspects for comparison should be available.

The great variety of workflow modeling methodologies shown by both systems makes the comparison of the process meta model a lot more difficult than the comparison of the organisational meta models. Therefore, an enhancement of the evaluation with a catalogue of basic control flow elements seems to be useful. The results of such an enhancement are a starting point for the evaluation of the semantic modeling power of different workflow management systems.

\section{REFERENCE META MODELS AS A BENCHMARK FOR META DATA MODELS}

\section{An Organisational Reference Model for Workflow Systems}

The evaluation of the meta data models described above as well as the analysis of two additional workflow management systems led to the conclusion, that an independent benchmark had to be established in order to enable a more objective analysis of workflow systems.

The evaluated workflow systems showed substantial weaknesses regarding their organisational modeling component, whereas the process modeling capabilities were much more sophisticated. Therefore an organisational reference model has been developed, which serves as a benchmark for the evaluation of meta data models (fig. 5). This reference meta model includes several aspects which enhance the ideas found (or missed) in the systems analysed. The main objective during the development of the reference meta model was to obtain a maximum of flexibility in order to use the model for a broad variety of organisations. Consequently, the meta data models of existing workflow systems served as a starting point for the reference model, but it finally turned out that a flexible meta model could be designed with a relatively small number of entity and relationship types.

\section{Additional Entity Types}

Most workflow systems provide the modeller with a role concept for the modeling of organisational entities. Roles serve as a container for the qualifications an actor has to have in order to fulfil a given task. However, in real life organisations there is a difference between qualifications and competencies. While the former are attributes of a specific actor and cannot be removed (ie. "speaks Spanish"), the latter depend on the position the actor occupies in a given organisation (ie. "may sign orders over $\$ 50.000$ ") and can be assigned and removed. Consistently, the entity type role is subdivided into the two entity types qualification and competence.

The modeling of committees, expert groups and other temporary organisational units is enabled by two elements. First, the strict organisational hierarchies $[(0,1)-(0, n)]$ found in WorkParty and FlowMark are replaced by structures 
$[(0, n)-(0, m)]$, which allow the modeling of multidimensional relationships between roles, positions and organisational units. Secondly, the relationship between actors and organisational units is separated by the introduction of positions, which form the static organisational structure of a company. A person can occupy several organisational positions and can therefore be a member of several organisational units, those being temporary or permanent.

Since an organisational position serves as a placeholder for a specific person, the competencies related to a position should be assigned to the position itself instead to the person occupying it (although it is possible to assign competencies to a person directly as well). With the increasing size of a company, the number of similar positions is likely to increase. In this case position types serve as a template for positions (eg. "secretary") which can be refined in a position instance (eg. "secretary of Prof. Becker").

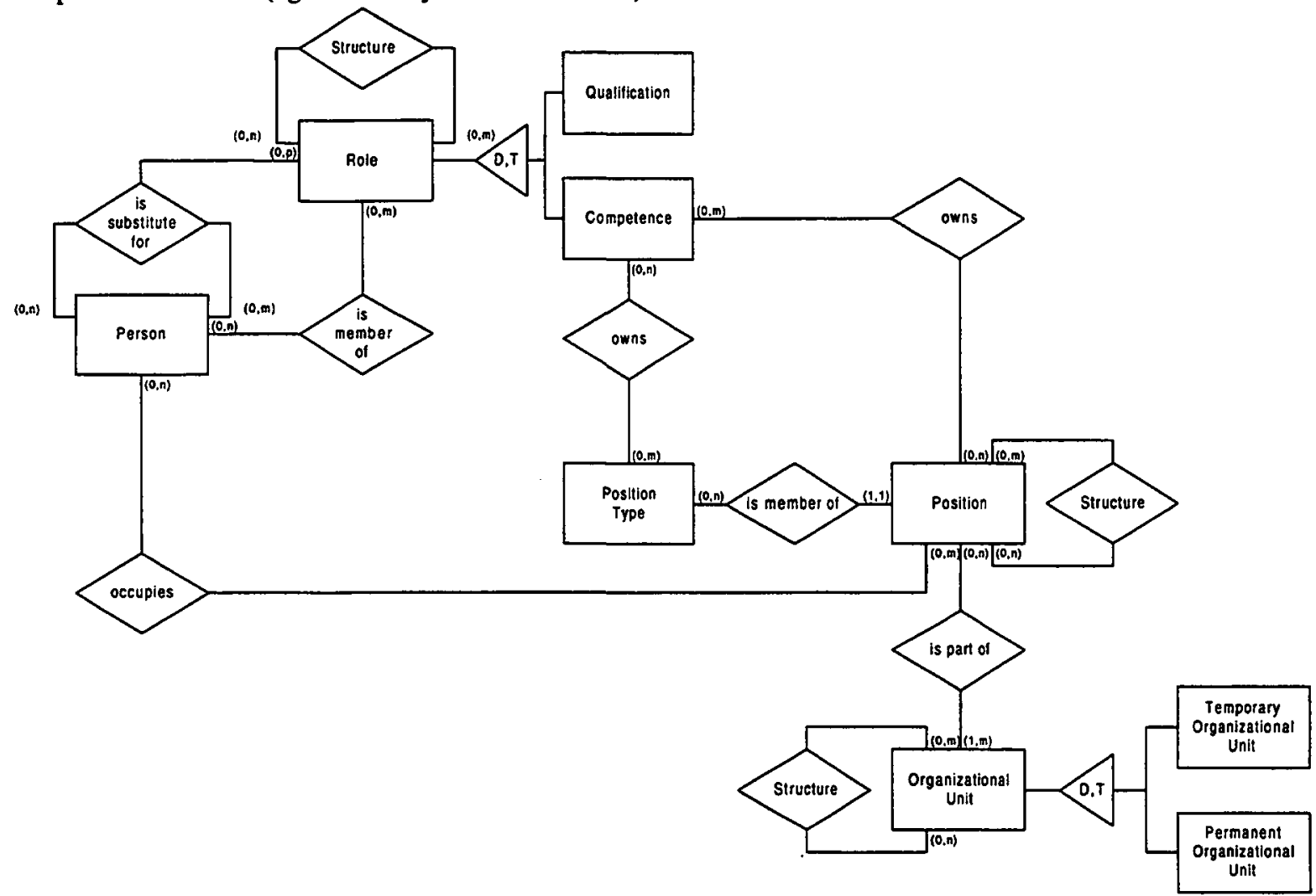

Fig. 5: Organisational reference meta model

\section{Substitute Relationships}

Not all workflow systems offer the possibilities of defining substitute relationships between employees. In most cases the assignment of an activity to a role instead of a specific person solves the problem of substituting an absent employee. In some cases (especially during an authorisation or an inspection activity) it is necessary to assign an activity to a specific person. Substitutes are necessary in these cases in order to preserve the flexibility of the original role system. Most substitute mechanisms allow an actor to inherit all qualifications of the original actor. In some systems (eg. WorkParty), the substitute mechanism relates two organisational positions instead of two actors. However, the complete inheritance of all rights can have serious impact on the security policies of an enterprise. Therefore it is desirable to be able to restrict a substitute relationship to specific roles of an actor. This is enabled by introducing a ternary relationship between the entity type person and the entity type role. A person can have several substitutes as well as be the substitute for several other people. The substitution may be reduced to certain roles of a person. Hence it is possible eg. to create a substitute for a manager that is not allowed to sign orders over $\$ 50.000$, because this specific role of the manager was excluded from the substitute relationship. 


\section{Process Dependent Staff Resolution}

During the instantiation of a workflow model it may be necessary to assign actors to activities depending on the state of process objects (eg. invoice, offer) or the state of activities already performed. It should therefore be possible to assign an activity not only to the entity types of an organisational meta model, but also to the relevant relationship types, such as "supervisor of the actor that performed the last activity". This option is useful in case of an exception, when an escalation mechanism redirects the current activity to the superior of the actor performing the activity. Another example of this mechanism is the double check of an invoice. In this situation it has to be made sure that the actor performing the second check is not the same actor that already performed the first check. For reasons of clarity, these relationships are not depicted in fig. 5 .

An important aspect of process dependent staff resolution is the use of process object attributes as variables for the activity definition, which is outlined by means of an example. During the auditing of accounts, it is necessary to model separate activities for every origin of a foreign bill (English, French, Spanish etc.) and to assign certain roles (Clerk with English language skills, Clerk with Spanish language skills etc.) to each of these activities. With the use of process objects it is possible to reduce the activities to one, where the origin of the bill itself serves as a variable for the role definition, ie. if an American bill is the process object of the current process instance, the required generic role ("X language skills") is replaced by a certain instance enhanced with the attributes of the process object ("English language skills"). Since it affects the interface between the meta process and the meta data model of a workflow management system, this aspect is not depicted in fig. 5 as well.

\section{Evaluating Individual Meta Models Against a Reference Model}

The comparison of the organisational meta models with the organisational reference model outlined above reveals some additional aspects. While the organisational meta model of WorkParty (fig. 1) shows entity types similar to those of the reference model (position type, organisational position etc.), the high number of relationship types tend to make the meta model unnecessarily complex. Contrary to this observation the entity types of the FlowMark meta model (fig. 2) show only little resemblance to those of the reference model. On the other hand some relationship types may be addressed directly in FlowMark (coordinates, is member of), which is not possible in WorkParty.

The use of a reference model helps directing the attention of the observer to aspects that would not have been analysed otherwise. Thus a reference model can improve the results of a meta model comparison. Reference meta models can serve as a foundation for the design of a process information system, used for process monitoring and controlling, as well as a guideline for information system designers deriving meta models from given systems.

\section{SUMMARY AND FUTURE DIRECTIONS}

A comparison of meta models can provide useful information for the evaluation of systems, but it depends on the context of the analysis. Organisational meta models of workflow management systems are limited to a well documented domain with little product-individual differences. In this context a meta model evaluation provides the analyst with important information about the modeling capabilities of the methods analysed. Process meta models, however, are only one instrument during the evaluation of workflow management systems. They are useful for the analyst to gain an overview about the modeling methodologies and to get acquainted with the individual systems, but they are not sufficient for a final decision for or against a specific system. The use of reference meta models, such as the organisational reference model can help the user find the aspects relevant to his decision.

Some extensions for existing modeling methodologies have been introduced, such as variable-based cardinalities, which enhance the suitability of these models. Future work should emphasise this aspect. Further fields of research are - besides the inclusion of other workflow management systems - the design of an inductively generated process reference meta model as well as the development of a catalogue of basic control flow elements.

\section{REFERENCES}

Batini, C., Ceri, S. \& Navathe, S. B. (1992) Conceptual Database Design, An Entity-Relationship-Approach, Redwood City et al.

Batini, C. \& Lenzerini, M. (1984) "A Methodology for Data Schema Integration in the Entity Relationship Model"” IEEE Transactions on Software Engineering, 6, Vol. 10, pp 650-664. 
Chen, P. P.-S. (1976) "The Entity-Relationship Model: Toward a Unified View of Data", ACM Transactions on Database Systems, 1, Vol 1, pp 9-36.

Derungs, M. (1997) Kundenorientierte Workflowprojekte, DUV, Wiesbaden.

Dunn, R. H. (1991) Software-Quality. Concepts and Plans, Englewood Cliffs.

Elmasri, R. \& Navathe, S. B. (1995) Fundamentals of Database Systems, $2^{\text {nd }}$ Ed., Benjamin/ Cummings Publishing.

Ferstl, O. K. \& Sinz, E. J. (1994) Grundlagen der Wirtschaftsinformatik. Band 1, $2^{\text {nd }}$ Ed., Vahlen (in German).

Galler, J. (1997) Vom Geschäftsprozeßmodell zum Workflow-Modell, Gabler, Wiesbaden (in German).

Georgakopoulos, D., Hornick, M. \& Sheth, A. (1995) "An Overview of Workflow-Management: From Process Modeling to Workflow Automation Infrastructure", Distributed and Parallel Databases, 2, Vol 3, pp 119 153.

Hars, A. (1994) Referenzdatenmodelle. Grundlagen effizienter Datenmodellierung, Gabler, Wiesbaden (in German).

IBM FlowMark Modeling Workflow. Version 2 Release 3. Document Number: SH19-8241-02, Ed.: IBM Germany, Wien.

Jablonski, St. \& Bussler, C. (1996) Workflow-Management: Modeling Concepts, Architecture and Implementation, International Thomson Computer Press.

Joosten, S. (1995) "Conceptual Theory for Workflow Management Support Systems". Technical Report. Centre for Telematics and Information Technology, University of Twente, Enschede.

Joosten, S. (1996) "WorkPAD: a Conceptual Framework for Process Analysis and Design". ACM Transactions on Office Information Systems (under submission).

Kazman, R., Bass, L., Abowd, G. \& Webb, M. (1994) "SAAM: A Method for Analyzing the Properties of Software Archietectures", Proceedings of the International Conference on Software Engineering - ICSE'16. Ed.: B. Fadini, Sorrento, pp 81-90.

Leymann, F. \& Altenhuber, W. (1994) "Managing business processes as information resources", IBM Systems Journal, 2, Vol 33, pp 326-348.

Nissen, H. W., Jeusfeld, M. A., Jarke, M., Zemanek, G. V. \& Huber, H. (1996) "Managing Multiple Requirements Perspectives with Metamodels", IEEE Software, 3, Vol 13, pp 37-48.

Rosemann, M. (1996) Komplexitätsmanagement in Prozeßmodellen, Gabler, Wiesbaden (in German).

Rupietta, W. (1997) Organisation and Role Models for Workflow Processes, Workflow Handbook 1997, Ed.: P. Lawrence, John Wilsey \& Sons Ltd, Chichester et al., pp 165-172.

Saeki, M. (1995) "Object-Oriented Meta Modelling", Proceedings of the $14^{\text {th }}$ int. Conference on Object Oriented and Entity Relationship Modelling, Ed.: M. P. Papazoglou, Berlin et al., pp 250-259.

Siemens Nixdorf (1995) Organisations- und Ressourcenmanagement, Version 2.0, Muenchen (in German).

Siemens Nixdorf (1994) WorkParty Benutzerhandbuch, Version 2.0, Muenchen (in German).

Steele, P. M. \& Zaslavsky, A. B. (1994) "The Role of Meta Models in Federating System Modelling Techniques", Proceedings of the $12^{\text {th }}$ International Conference on the Entity-Relationship Approach - ER '93, Eds.: R. A. Elmasri, V. Kouramajian, B. Thalheim. Berlin et al., pp 315-326.

WfMC (1996) Workflow Management Coalition Terminology \& Glossary. Document Nr. WFMC-TC-1011, $2^{\text {nd }}$ ed., Bruessel. 